\title{
A NEW APPROACH IN COMBINED MODELING OF MRI AND BLOOD FLOW: A PRELIMINARY STUDY
}

\author{
Krzysztof Jurczuk ${ }^{1,2}$, Marek Kretowski ${ }^{2}$, Pierre-Anatoine Eliat ${ }^{3}$, Jean-Jacques Bellanger ${ }^{1}$, \\ Hervé Saint-Jalmes ${ }^{1}$, Johanne Bézy-Wendling ${ }^{1}$ \\ ${ }^{1}$ INSERM U642, Laboratoire Traitement du Signal et de l'Image, Rennes, France. \\ Université de Rennes 1, LTSI, Campus de Beaulieu, Rennes 35042 Cedex, France. \\ ${ }^{2}$ Faculty of Computer Science, Bialystok University of Technology, Wiejska 45a, 15-351 Bialystok, Poland. \\ ${ }^{3}$ PRISM-Villejean - IFR 140 - Biogenouest, Université de Rennes, Rennes, France.
}

\begin{abstract}
We propose a computational model to simulate the physical processes implied in the Magnetic Resonance Images formation in vascularised tissues. A combined model of MRI acquisition and blood flow is presented. The blood flow patterns are modeled using the Lattice Boltzmann Method, and the magnetic resonance experiments follow the Bloch equation. A new algorithm has been developed to compute the local magnetizations transport during the excitation, precession and relaxation steps. First results were compared to theoretical values of phase flow accumulation and amplitude attenuation. Next, the proposed algorithm is applied to simulate MR images in a simple stenosed vessel and in bifurcations. Characteristic flow artefacts are observed.
\end{abstract}

Keywords: Biological system modeling, computer simulation, magnetic resonance imaging, fluid flow, Lattice Boltzmann method.

\section{INTRODUCTION}

Computational models are very useful to experiment some modifications that can not be easily applied to real systems. For instance, structural or functional properties of tissues or organs, at different scales, can often not be directly changed on animals or patients. The interest of models also appears when the number of scenarios to be tested is important, which would imply numerous expensive experiments. These two situations are encountered when the vascular changes related to the development of certain pathologies, like tumours, have to be studied in medical imaging. Detection and characterization of early image modifications, reflecting physiological disorders, benefit of models.

In order to reach this goal, it is necessary to model not only the physiological perturbations but also the physical phenomena involved in the image formation. We propose, in this preliminary study, to couple a vascular model where flows are simulated by the Lattice Boltzmann Method (LBM) [1], and a model of Magnetic Resonance Image (MRI) acquisition, based on the resolution of the Bloch equation. The main challenge in this coupled model is to consider the nuclei magnetization transport during nuclear magnetic resonance imaging experiments (including excitation, precession, relaxation as well as space encoding and signal sampling processes).

In our model, a set of quadrangles (equivalent to sub-voxels) is used to represent the vascularized three dimensional region that has to be imaged. To each quadrangle, magnetic characteristics are assigned (proton density, relaxation times), as well as flow properties, i.e. velocity and direction of the fluid filling it.
During the imaging simulation, the local magnetizations are modified according to the magnetic resonance (MR) phenomenon and flow in parallel. The flow influence is taken into account by a newly proposed transport magnetization algorithm based on the Eulerian coordinates approach (i.e. stationary frame) [2], while MR processes are modeled by the discrete time solution of the Bloch equation in the form of rotation matrices and exponential scaling [3]. In section 2, the LBM model is described and simulated results are presented, in simple vascular geometries. Section 3 is aimed at presenting the MR image formation first for a static tissue, and then for a mixture of static and dynamic spins (these ones moving because of blood flow). Preliminary results of simulated images are shown for two typical MRI sequences. Then, the validation process based on phase and amplitude magnetization evolution over time is also explained. Future works of modeling of MRI with flow are finally put in perspectives.

Other MRI flow simulators can be found in the literature. They can be classified into two groups: Eulearian based approaches [4], [5] and Lagrangian based ones [6], [7]. The Lagrangian based solutions are known to be less accurate to simulate MRI of flow in complex geometries because of difficulties of tracking spin trajectories near walls. On the other side, all stationary frame solutions integrate the Bloch equation with an additional transport term. As a result, voxel intensities are directly obtained (not a magnetic signal like in a real equipment) and in order to take into account time-dependent flow artefacts, additional mechanisms have to used, e.g. phase shift correction etc. In our algorithm, the LBM is used for the first time, which can provide easier and quicker fluid dynamics simulations. Furthermore, we applied the discrete-event Bloch equation resolution which closely follows the physical process of MRI and, in combination with the new magnetization transport algorithm, naturally incorporates flow artefacts (no additional numerical corrections are required).

\section{MODELING OF FLOW WITH LBM}

\subsection{Method}

The Lattice Boltzmann Model [1] is a modern Computational Fluid Dynamics method used to numerically solve the Navier Stokes equations. It is based on the evolution of statistical fluid distribution on a lattice/grid (inspired by kinetic theory). The fluid characteristics evolve on the grid nodes, according to rules at the mesoscopic level providing that mass and momentum conservation laws at the macroscopic level are fulfilled. Concerning our modeling task, LBM presents the advantages of $i$ ) being usable in complex computation domains (like complete vascular networks, complex boundary conditions), ii) using relatively low computational load and iii) 
facilitating the computation parallelisation [8]. All these advantages make this method ideal for an easy and fast computation of the fluid flow characteristics which then can be used to simulate the combined MRI and flow processes.

In this method, fluids are considered as a set of numerous virtual particles whose movements over the grid of nodes are described by statistical laws. Two processes intervene in the particles displacements: the propagation (which suppose that the particles move without encountering others particles) and the collision between particles. The mathematical expression of these two actions is given by the discrete Boltzmann equation:

$$
f_{i}\left(\mathbf{r}+\mathbf{e}_{i} \Delta t, t+\Delta t\right)-f_{i}(\mathbf{r}, t)+F=\Omega
$$

Where $f_{i}$ is the value of the distribution function of particles leaving the grid node situated at $\mathbf{r}$ position in the discrete $i$ direction with the discrete $\mathbf{e}_{i}$ velocity in the discrete time $\Delta t ; F$ is the external force, and $\Omega$ is the collision operator.

The Bhatnagar-Gross-Krook collision method was used to approximate the collistion operator. It introduces a relaxation term (related to viscosity) and provides mass and momentum conservation laws to be verified [8]. In two dimensional flows we use the D2Q9 model (9 discrete velocities), while in three dimensional simulations the D3Q19 model was chosen.

\subsection{Results of simulation in simple geometries}

Fig. 1 shows the comparison of analytical solving of Poiseuille equation (red) with LBM simulation (black) in a $3 \mathrm{D}$ straight vessel (only one section is presented). Successive curves show the convergency process of LBM solution to the analytical one (starting from top-left side, in the right direction and in the subsequent rows, ending on bottom-right side).

First, the LBM model has been applied to a simple 2D vessel presenting a stenosis, later to a vascular tree with 3 bifurcations (Fig. 2). The flow was driven by a pressure difference. Even a visual image inspection shows that satisfactory flow patterns were obtained.

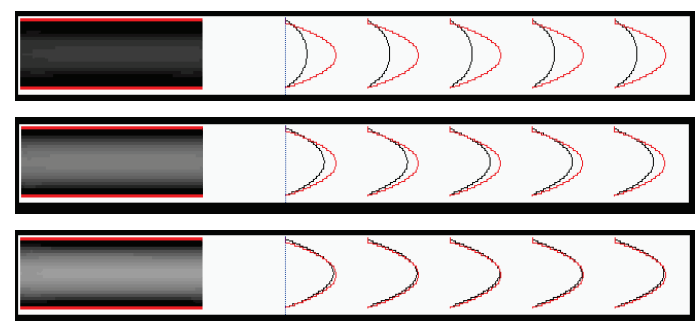

Figure 1: Comparison of analytical solving of Poiseuille equation (red) with LBM simulation (black), in a 3D vessel.

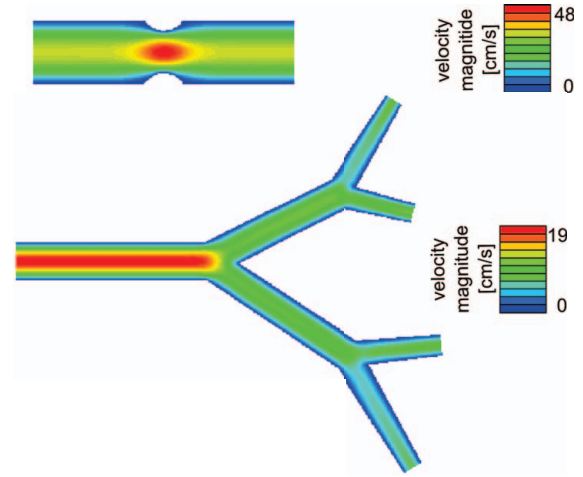

Figure 2: Images of the simulated velocity magnitude of the flow in two 2D vascular structures: (top) a simple stenosed vessel and (bottom) a vascular tree with 3 bifurcations.

\section{MODELING OF MRI ACQUISITION}

\subsection{General approach}

Based on the LBM calculation, at each grid node (regularly filling the grid), the mean macroscopic velocity and direction of fluid is known, i.e. the three components of the velocity vector.

The area of interest is divided into quadrangles and the grid node is situated in the centre of each quadrangle (grid node where LBM calculations were done). In each quadrangle, the local magnetization is followed in discrete moments taking into account both magnetization transport and magnetic resonance imaging processes (excitation, relaxation, etc.), according to the following equation:

$\mathbf{M}(\mathbf{r}, t+\Delta t)=\mathbf{M}_{M R I}(\mathbf{r}, \Delta t)\left(\mathbf{M}(\mathbf{r}, t)+\Delta \mathbf{M}_{F L O W}(\mathbf{r}, \Delta t)\right)$ Where $\mathbf{M}_{M R I}$ is the influence of the nuclear magnetic resonance phenomena during the $\Delta t$ period, $\Delta \mathbf{M}_{F L O W}$ is the flow influence during $\Delta t$ period and $\mathbf{r}=(x, y, z)$ is the spatial coordinates of the considered quadrangle.

\subsection{MRI model without flow}

The influence of nuclear magnetic resonance phenomena during imaging sequence $\left(\mathbf{M}_{M R I}\right)$ is modeled by the Bloch equation which enables us to follow the magnetization $(\mathbf{M})$ changes in time $(t)$ :

$$
\frac{d \mathbf{M}(t)}{d t}=\gamma(\mathbf{M}(t) \times \mathbf{B}(t))-\left(\begin{array}{c}
M_{x}(t) / T_{2} \\
M_{y}(t) / T_{2} \\
\left(M_{z}(t)-M_{0}\right) / T_{1}
\end{array}\right)
$$

Where $T_{1}$ and $T_{2}$ are the relaxation times, $\mathrm{M}_{0}$ is the equilibrium magnetization, $\mathbf{B}$ is the applied magnetic field and $\gamma$ is the gyromagnetic ratio. In order to follow closely MRI process, we use the discrete-event solution of the Bloch equation [3]. Thus, in each quadrangle, the magnetization evolution is iteratively computed according the following equations:

$$
\begin{gathered}
\mathbf{M}(\mathbf{r}, t+\Delta t)=\mathbf{M}_{M R I}(\mathbf{r}, \Delta t) \mathbf{M}(\mathbf{r}, t) \\
\mathbf{M}_{M R I}=\mathbf{E}_{R E L A X} \mathbf{R}_{Z}(Q) \mathbf{R}_{R F}
\end{gathered}
$$

Where the $\mathbf{E}_{R E L A X}$ matrix provides the relaxation effects (transverse exponential decay and longitudinal exponential increase of the magnetization vector), $\mathbf{R}_{Z}(Q)$ is the rotation matrix of angle $Q$ around $z$ axis resulting from the applied magnetic gradients and $\mathbf{R}_{R F}$ is the rotation matrix responsible for the excitation process due to the applied RF pulse. During the signal acquisition process, the contribution of all quadrangles is summed and saved in the k-space matrix which is next Fourier transformed to yield an image. The size of the image voxel cannot be less than the size of a quadrangle, i.e. intensity of a single voxel comes from at least one quadrangle.

\subsection{Transport of magnetization}

The solution of MRI of flow couples the described above MRI model for stationary tissue and the magnetization transport algorithm. As a result, we are able to follow the nuclei magnetization transport during nuclear magnetic resonance imaging simulations.

Each quadrangle contains information necessary to compute a local spin magnetization, i.e. proton density, spin-lattice relaxation time $\left(T_{1}\right)$ and spin-spin relaxation time $\left(T_{2}\right)$. Moreover, values of flow direction and velocity are also assigned to each quadrangle after computation by LBM (see $\S 2$ ). For stationary tissues (e.g. vessel walls, parenchyma, etc.), the flow velocity is considered to be zero. 


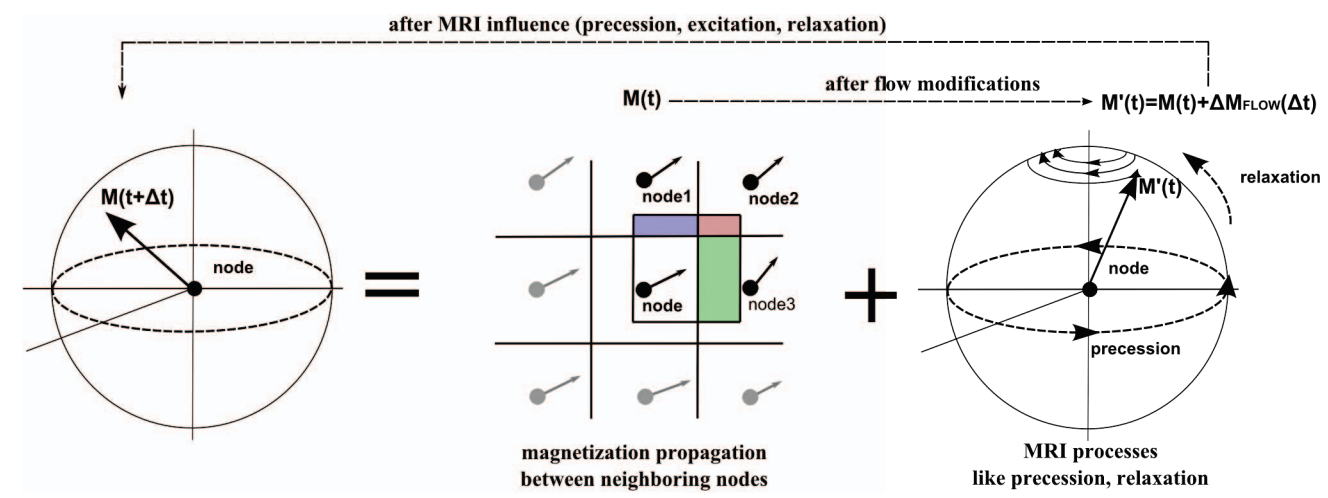

Figure 3: Method to couple the transport of local magnetizations and MRI processes. $M(t)$ is the initial magnetization in time $t$, $\mathrm{M}^{\prime}(\mathrm{t})=\mathrm{M}(\mathrm{t})+\Delta \mathrm{M}_{\mathrm{FLOW}}(\Delta \mathrm{t})$ is the magnetization after changes due to flow and $\mathrm{M}(\mathrm{t}+\Delta \mathrm{t})$ is the magnetization after $\Delta \mathrm{t}$ time taking into account flow and magnetic resonance phenomena (e.g. relaxation, precession). The flow influence is shown in the middle part of the figure. The green, pink and violet rectangles represent the magnetization fractions that flow out from the quadrangle.

The whole MRI simulation is divided into sufficiently small time steps $(\Delta t)$ (the time step is smaller than the shortest time needed by the fluid to go from one lattice node to the other). At each time step, in each quadrangle, the new value of magnetization is calculated $(\mathbf{M}(t+\Delta t))$ (see Fig. 3). The first step consists in computing the flow influence $\left(\Delta \mathbf{M}_{F L O W}\right)$ (middle part of Fig. 3). Based on the velocity values and the flow direction, in each quadrangle the magnetization fractions are propagated to the neighboring quadrangles. Thus, part of the magnetization goes out from the quadrangle (rectangles filled in green, pink and violet colors). In the same time, magnetization fractions also enter the considered quadrangle by coming from neighboring quadrangles.

These operations allow us to simulate the transport of magnetizations or, in other words, the flow of excited hydrogen atoms. Next, the influence of nuclear magnetic resonance phenomena is taken into account $\left(\mathbf{M}_{M R I}\right)$, i.e. excitation, precession, relaxation (right part of Fig. 3).

\subsection{Results}

Preliminary results of MR simulated images are presented in Figure 4. Two MR sequences have been reproduced: spin echo (SE), and gradient echo (GE).

In all images, characteristic flow artefacts are observed. For the single vessel, in both images, near the stenosis the signal

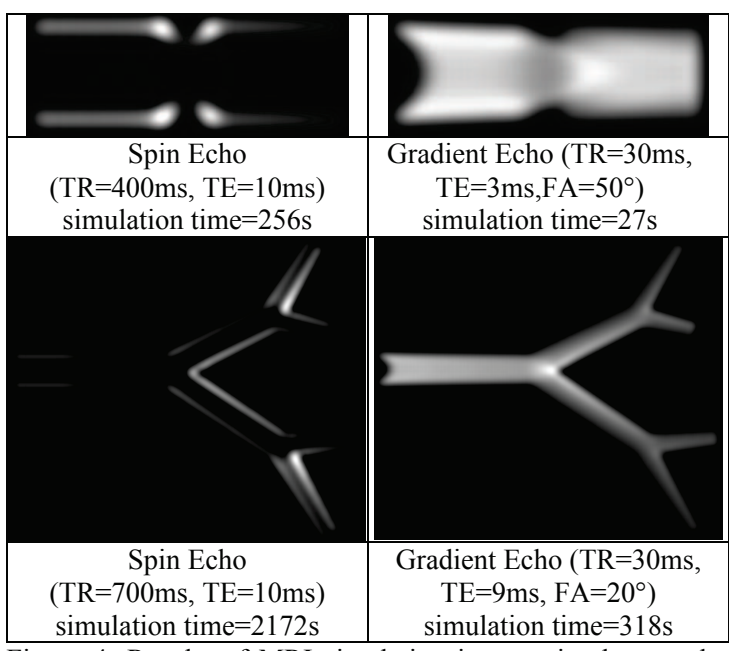

Figure 4: Results of MRI simulation in two simple vascular geometries: (top) a 2D tube, (bottom) a 3 bifurcation tree. enhancement is clearly visible (first row images). It results from the phase misregistration (phase shift) effect that causes the displacement artefact due to time interval between phase and frequency encoding processes [9]. All the vascular tree images show also this artefact, i.e. the lumen of diagonal vessels is displaced to the right (enhacement of inside vessel walls). Moreover, GE images (right column), illustrate the inflow effect as a result of entering the fresh blood to the vessel from the left side. Another type of flow artefacts is visible in all SE images. Mainly the slowest flowing particles (i.e. close to walls) provide signal. It comes from gradually losing phase coherence by flow particles. It is also worth noting that simulation time needed to obtain images is relatively short.

\section{VALIDATION}

Two main kinds of measures have been done to evaluate our model. First, considering objects without flow, we compared the amplitude and phase of the signal obtained with our method, with the ones generated by the SIMRI simulator [10]. A lot of 1D and 2D virtual objects have been tested. In all cases, an excellent agreement (mean percentage difference less than 5\%) was obtained. The amplitude and phase of the acquired signal from one particular 1D object are presented in Fig. 5, while Table 1 resumes the object characteristics.

Then we studied the amplitude and phase of the signal obtained from a rigid tube with different values of flow (Fig. 6). The presented values of phase and amplitude were measured in the isocenter of magnetic field for one quadrangle. These values compare well with theoretical ones, obtained from the magnetic resonance angiography investigation conducted by Nishimura [11] and allowed us to check the influence of flow on dephasing.

\begin{tabular}{|c|c|c|}
\hline points & $0-63$ & $64-127$ \\
\hline proton density & \multicolumn{1}{|c|}{ linearly from 1 to 100} \\
\hline T1 & \multicolumn{1}{|c|}{ linearly from 100 to 1} \\
\hline T2 & 100 & 50 \\
\hline
\end{tabular}

Table 1: 1D object (128 points, 1 meter) used to compare the simulation results of our model with the SIMRI [10].

When there is no flow, at time TE, all spins have the same phase (they are rephased), usually equal to 0 . Thus, the received signal is the sum of all amplitudes and is high (no influence of dephasing).

In the case of a non-zero flow, an accumulation of phase differences is observed (spins lose or gain phase) because they 
are submitted to different amplitudes of magnetic gradients, depending on their positions. Spins can then have different phases, and this dephasing process leads to a smaller (even nul) signal. Moreover, our validation included different kinds of RF pulses (e.g. rectangular, sinc, Gaussian) and other imaging sequences, which are not represented here.
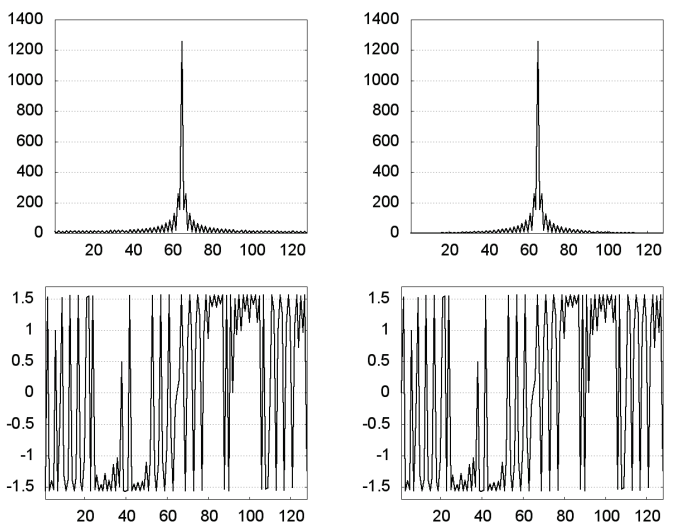

Figure 5: Amplitude (top) and phase (bottom) of the signal received with a spin echo sequence $(\mathrm{TR}=1000 \mathrm{~ms}$ and $\mathrm{TE}=100$ $\mathrm{ms})$ : left - with our method, right - with SIMRI [10].

\section{CONCLUSION / PERSPECTIVES}

We proposed a new method of modeling MR images with flow. This method is based on a combination of $i$ ) flow computation by LBM, ii) a new magnetization transport algorithm and iii) a MR simulation based on following the magnetization in time, applying a set of several matrices, each one representing the effect of a MR phenomenon (gradient, RF pulse, relaxation). The good preliminary results obtained in $2 \mathrm{D}$ on simple vascular structures as well as the small computation times encourage us to develop our simultor in several ways: more complex vascular networks, slice selection, flow compensation sequences. A step of validation by comparison with real images has also been started and will be completed in future works. Parallelisation of the computations has already been introduced and will be still more developped in the next version of our combined model, at each step (flow calculation, magnetization transport and MR acquisition)

\section{REFERENCES}

[1] S. Succi, The lattice Boltzmann equation for fluid dynamics and beyond, Clarendon Press, Oxford, 2001.

[2] D. J. Tritton, Physical fluid dynamics, Oxford University Press, Oxford, 1988.

[3] J. Bittoun, J. Taquin, M. Sauzade, A computer algorithm for the simulation of any nuclear magnetic resonance (NMR) imaging method, Magnetic Resonance Imaging, vol. 3, pp. 363-376, 1984

[4] L.D. Jou, D. Saloner, A numerical study of magnetic resonance images of pulsatile flow in a two dimensional carotid bifurcation: a numerical study of MR images, Medical Engineering \& Physics, vol. 20(9), pp. 643-652, 1998.

[5] S. Lorthois, J. Stroud-Rossman, S. Berger, L.D. Jou, D. Saloner, Numerical simulation of magnetic resonance angiographies of an anatomically realistic stenotic carotid bifurcation, Annals of Biomedical Engineering, vol. 33(3), pp. 270-283, 2005.

[6] R. van Tyen, D. Saloner, L.D. Jou, S. Berger, MR imaging of flow through tortuous vessels: a numerical simulation, Magetic Resonance in Medicine vol. 31(2), pp. 184-195, 1994.

[7] I. Marshall, Computational simulations and experimental studies of 3D phase-contrast imaging of fluid flow in carotid bifurcation geometries, Journal of Magnetic Resonance Imaging, vol. 31(4), pp. 928-934, 2010.

[8] D.A. Wolf-Gladrow, Lattice-gas cellular automata and lattice Boltzmann models, Springer-Verlang, Berlin, 2000.

[9] H. Bosman, R. Hausmann, Flow-dependant acquisition techniques. In: I.P. Arlat, G. Bongartz, G. Marchai (ed.), Magnetic Resonance Angiography, Springer-Verlang, Berlin, 2002.

[10] H. Benoit-Cattin, G. Collewet, B. Belaroussi, H. SaintJalmes, C. Odet, The SIMRI project: A versatile and interactive MRI simulator, Journal of Magnetic. Resonance, vol. 173, pp. 97-115, 2005.

[11] D.G. Nishimura, A. Macovski, J.M. Pauly, Magnetic resonance angiography, IEEE Transactions on Medical Imaging, vol. 5(3):140-151, 1986.
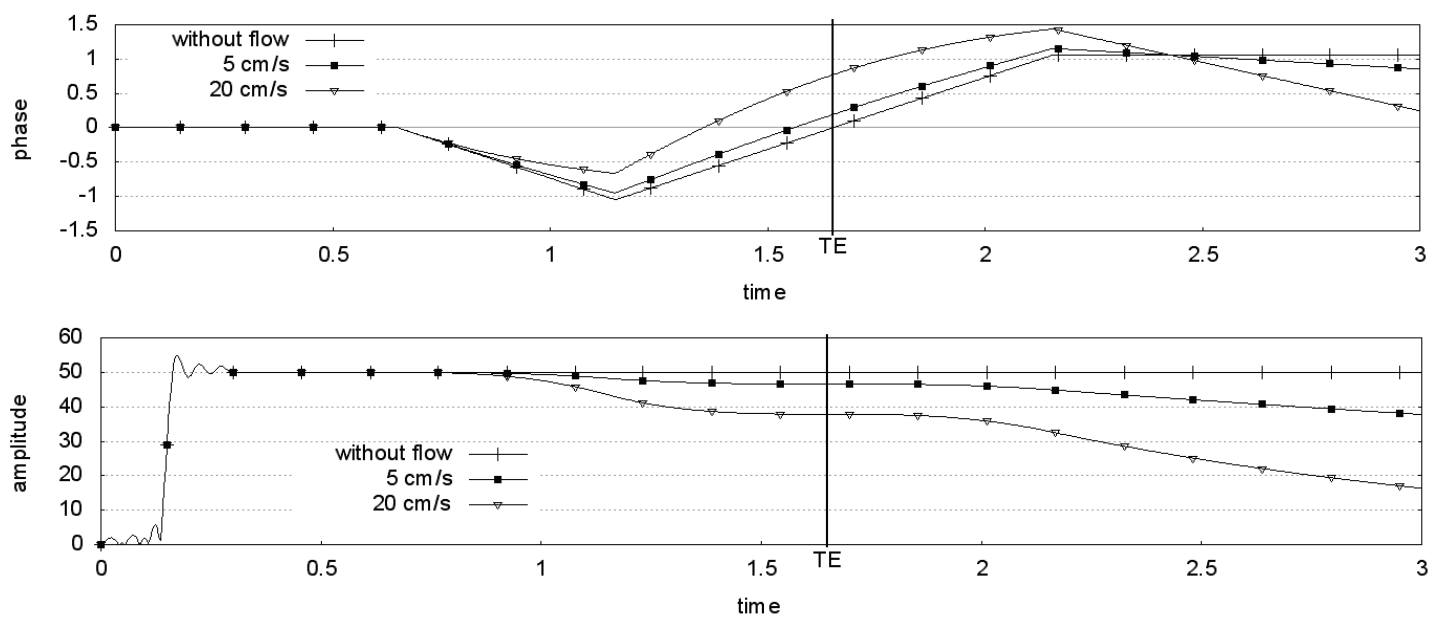

Figure 6: Illustration of the influence of dephasing caused by flow, on phase, and amplitude. Two values of flow and no flow are considered $(0,05 \mathrm{~cm} / \mathrm{s}, 20 \mathrm{~cm} / \mathrm{s})\left(\mathrm{TE}=1.5 \mathrm{~ms}\right.$, acquisition time $=1 \mathrm{~ms}$, flip angle $=30^{\circ}$, sinc shape $\mathrm{RF}$ pulse time $\left.=0.3 \mathrm{~ms}\right)$.

This work was supported in part by the Region Bretagne in France and in part by Bialystok University of Technology under Grant W/WI/3/11. 